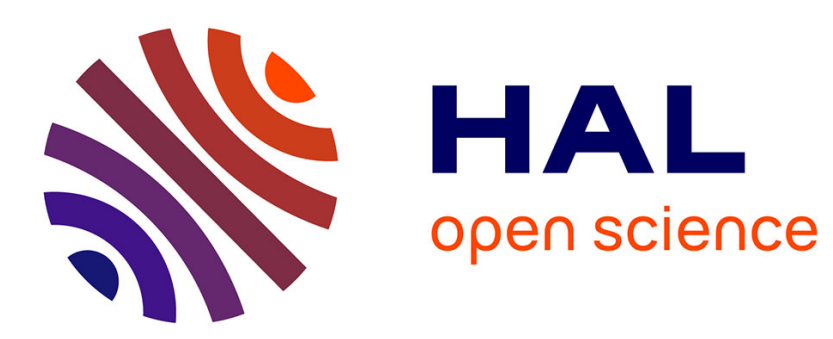

\title{
Magnetic Field Produced by a Tile Permanent Magnet Whose Polarization is both Uniform and Tangential
}

Romain Ravaud, Guy Lemarquand, Valérie Lemarquand, Claude L. Depollier

\section{To cite this version:}

Romain Ravaud, Guy Lemarquand, Valérie Lemarquand, Claude L. Depollier. Magnetic Field Produced by a Tile Permanent Magnet Whose Polarization is both Uniform and Tangential. Progress In Electromagnetics Research B, 2009, 13, pp.1-20. 10.2528/PIERB08121901 . hal-00360679

\section{HAL Id: hal-00360679 https://hal.science/hal-00360679}

Submitted on 11 Feb 2009

HAL is a multi-disciplinary open access archive for the deposit and dissemination of scientific research documents, whether they are published or not. The documents may come from teaching and research institutions in France or abroad, or from public or private research centers.
L'archive ouverte pluridisciplinaire HAL, est destinée au dépôt et à la diffusion de documents scientifiques de niveau recherche, publiés ou non, émanant des établissements d'enseignement et de recherche français ou étrangers, des laboratoires publics ou privés. 
Progress In Electromagnetics Research B, Vol. 13, 1-20, 2009

\title{
MAGNETIC FIELD PRODUCED BY A TILE PERMANENT MAGNET WHOSE POLARIZATION IS BOTH UNIFORM AND TANGENTIAL
}

\author{
R. Ravaud, G. Lemarquand, V. Lemarquand \\ and C. Depollier
}

Laboratoire d'Acoustique de l'Universite du Maine, UMR CNRS 6613 Avenue Olivier Messiaen, 72085 Le Mans, France

\begin{abstract}
This paper presents the exact 3D calculation of the magnetic field produced by a tile permanent magnet whose polarization is both tangential and uniform. Such a calculation is useful for optimizing magnetic couplings or for calculating the magnetic field produced by alternate magnet structures. For example, our 3D expressions can be used for calculating the magnetic field produced by a Halbach structure. All our expressions are determined by using the coulombian model. This exact analytical approach has always proved its accuracy and its usefulness. As a consequence, the tile permanent magnet considered is represented by using the fictitious magnetic pole densities that are located on the faces of the magnet. In addition, no simplifying assumptions are taken into account for calculating the three magnetic field components. Moreover, it is emphasized that the magnetic field expressions are fully three-dimensional. Consequently, the expressions obtained are valid inside and outside of the tile permanent magnet, whatever its dimensions. Such an approach allows us to realize easily parametric studies.
\end{abstract}

\section{INTRODUCTION}

The three-dimensional analytical calculation of the magnetic field produced by permanent magnets is very useful for optimizing alternate magnet structures or magnetic couplings. A previous paper written by the authors gave the expressions of the magnetic field created by a tile permanent magnet radially magnetized [1]. We use the same approach in this paper for calculating the magnetic field produced by

Corresponding author: G. Lemarquand (guy.lemarquand@univ-lemans.fr). 
a tile permanent magnet whose polarization is both tangential and uniform. To our knowledge, this calculation has never been carried out by other authors. However, the analytical method for obtaining the magnetic field expressions has been used by other authors interested in the analytical expressions of the magnetic field produced by arcshaped permanent magnets $[2-7]$. The first studies dealing with the three-dimensional calculation of magnetic fields created by arc-shaped permanent magnets used semi-analytical expressions that were based on one, two or three numerical integrations. However, these approaches are more accurate than finite-element methods [8-10]. Other papers have proved the usefulness of three-dimensional analytical approaches [12-15]. We can say that it is always more interesting to have an exact analytical expression rather than a numerical calculation for calculating the magnetic field produced by a permanent magnet [1618]. Authors generally use the Green's function or the Coulombian model for determining the magnetic field created by a tile permanent magnet [19-21]. However, they often neglect the curvature of the magnets in order to simplify the expressions $[22,33]$. In addition, no authors have determined the exact expressions of the magnetic field produced by a tile permanent magnet whose polarization is both uniform and tangential. However, such tile permanent magnets are commonly used in Halbach structures [34] (Fig. 1), in alternate magnet structures (Fig. 2) or in magnetic couplings in which the calculation of the torque between two rotors are required. This paper gives the three components of the magnetic field produced by a tile permanent magnet whose polarization is both uniform and tangential. Our exact approach is based on the Coulombian model. The interest of using

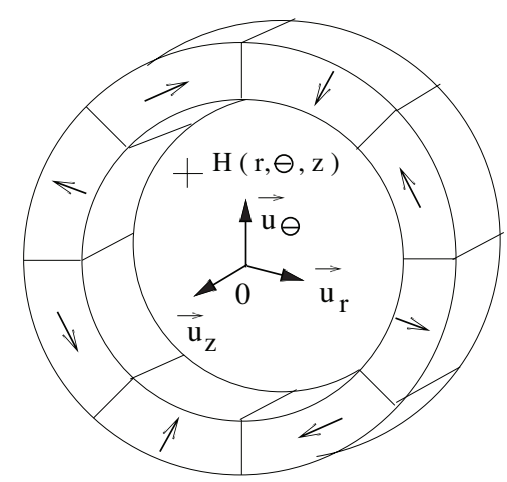

Figure 1. Representation of a Halbach structure: an assembly of tile permanent magnets whose polarizations are radial and tangential. 


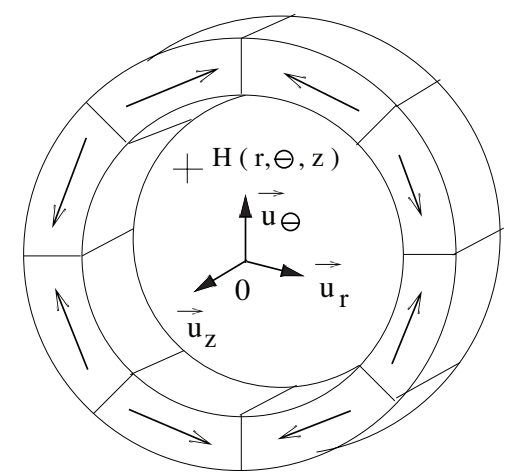

Figure 2. Representation of an alternate magnet structure: an assembly of tile permanent magnets whose polarizations are both uniform and tangential.

analytical approaches is mainly due to their accuracy, their very low computational cost and their easiness. Indeed, such approaches allow us to realize easily parametric studies. In addition, it is noted that no simplifying assumptions are done for these calculations. This is an important point because even if $2 \mathrm{D}$ models can be used for calculating the magnetic field created by such structures, some effects cannot be predicted by these models and the accuracy of the results can be questionable. However, the 2D approaches are fully analytical and their computational cost is thus very low. Furthermore, it is emphasized that the magnetic field expressions are fully threedimensional. Consequently, the expressions obtained are valid inside and outside of the tile permanent magnet, whatever its dimensions. The effects of all the magnetic pole contributions are discussed. We can say that such structures are interesting because they offer new prospects in the production of magnetic field. All the expressions determined in this paper are available online [35].

\section{NOTATION AND GEOMETRY}

The geometry considered and the related parameters are shown in Fig. 3. The tile inner radius is $r_{1}$, the tile outer radius is $r_{2}$, its angular width is $\theta_{2}-\theta_{1}$ and its height is $z_{2}-z_{1}$. The magnetic polarization of the tile is both tangential and uniform. This means that the magnetization direction is the same in the whole tile and is the tangent to the tile arc in its middle. Such a polarization is voluntarily chosen because such a tile permanent magnet can be used for enhancing 


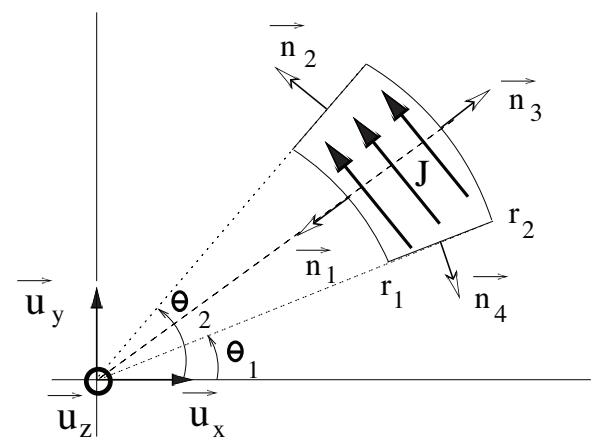

Figure 3. Representation of the geometry considered. The tile inner radius is $r_{1}$, the tile outer radius is $r_{2}$, its angular width is $\theta_{2}-\theta_{1}$, its magnetic polarization is $\vec{J}$.

the magnetic field inside an ironless motor using arc-shaped permanent magnets. Indeed, the assembly of several tile permanent magnets with several magnetizations allow us to obtain great magnetic fields.

Several approaches are generally used for determining the magnetic field components created by tile permanent magnets (Colombian Model, Green's functions, two-dimensional approach, finite-element method). The Colombian model is used in this paper. The main reason lies in the fact that analytical expressions determined with this model allow us to realize easily parametric studies. Therefore, we must take into account the magnetic pole surface densities located on all the faces of the tile permanent magnet. It is noted that no magnetic pole volume densities appear in this configuration because the polarization is uniform. However, as this polarization is rather non-conventional, we present in the next section a method to determine precisely where the magnetic fictitious pole appear on the tile permanent magnet faces.

\section{STUDY OF THE REPARTITION OF THE SURFACE MAGNETIC POLE DENSITIES}

\subsection{Obtaining the Magnetic Pole Surface Densities}

According to the Colombian model, the magnetic pole surface densities can be determined by calculating the scalar product between the polarization vector $\vec{J}$ and the four normal units $\vec{n}_{1}, \vec{n}_{2}, \vec{n}_{3}$ and $\vec{n}_{4}$. Let us first define the vector polarization $\vec{J}$. As the polarization considered is uniform and tangential, the vector polarization $\vec{J}$ is expressed as 
follows:

$$
\vec{J}=-J \sin \left(\frac{\theta_{2}+\theta_{1}}{2}\right) \vec{u}_{x}+J \cos \left(\frac{\theta_{2}+\theta_{1}}{2}\right) \vec{u}_{y}
$$

The four normal units are defined as follows:

$$
\begin{aligned}
& \vec{n}_{1}=-\cos (\theta) \vec{u}_{x}-\sin (\theta) \vec{u}_{y} \\
& \vec{n}_{2}=-\sin \left(\theta_{2}\right) \vec{u}_{x}+\cos \left(\theta_{2}\right) \vec{u}_{y} \\
& \vec{n}_{3}=+\cos (\theta) \vec{u}_{x}+\sin (\theta) \vec{u}_{y} \\
& \vec{n}_{4}=+\sin \left(\theta_{1}\right) \vec{u}_{x}-\cos \left(\theta_{1}\right) \vec{u}_{y}
\end{aligned}
$$

Consequently, we deduct that the magnetic pole surface densities are the following:

$$
\begin{aligned}
\sigma_{1}^{*}(\theta) & =-J \sin \left(\theta-\left(\frac{\theta_{1}+\theta_{2}}{2}\right)\right) \\
\sigma_{2}^{*} & =J \cos \left(\frac{\theta_{1}-\theta_{2}}{2}\right) \\
\sigma_{3}^{*}(\theta) & =J \sin \left(\theta-\left(\frac{\theta_{1}+\theta_{2}}{2}\right)\right) \\
\sigma_{4}^{*} & =-J \cos \left(\frac{\theta_{1}-\theta_{2}}{2}\right)
\end{aligned}
$$

\subsection{Analysis of the Repartition of the Magnetic Pole Surface Densities}

The magnetic pole surface densities located on the inner and outer faces of the tile permanent magnet $\sigma_{1}^{*}$ and $\sigma_{3}^{*}$ are not constant but depend on the angle $\theta$. A careful study shows that the sign of the magnetic pole surface density varies on the face whose normal unit is $\vec{n}_{1}$ and the face whose normal unit is $\vec{n}_{3}$. Indeed, let us first consider the face whose normal unit is $\vec{n}_{1}$. In this surface, the magnetic pole surface density is defined by $-J \sin \left(\theta-\left(\frac{\theta_{1}+\theta_{2}}{2}\right)\right)$. Therefore:

$$
\begin{aligned}
& \theta_{1} \leq \theta<\left(\frac{\theta_{1}+\theta_{2}}{2}\right) \Rightarrow \sigma_{1}(\theta)^{*}>0 \\
& \theta=\left(\frac{\theta_{1}+\theta_{2}}{2}\right) \Rightarrow \sigma_{1}(\theta)^{*}=0 \\
& \left(\frac{\theta_{1}+\theta_{2}}{2}\right) \leq \theta<\theta_{2} \Rightarrow \sigma_{1}(\theta)^{*}<0
\end{aligned}
$$


Let us now consider the face whose normal unit is $\vec{n}_{3}$ in which the magnetic pole surface density is defined by $J \sin \left(\theta-\left(\frac{\theta_{1}+\theta_{2}}{2}\right)\right)$. We deduct the following relations:

$$
\begin{aligned}
& \theta_{1} \leq \theta<\left(\frac{\theta_{1}+\theta_{2}}{2}\right) \Rightarrow \sigma_{3}(\theta)^{*}<0 \\
& \theta=\left(\frac{\theta_{1}+\theta_{2}}{2}\right) \Rightarrow \sigma_{3}(\theta)^{*}=0 \\
& \left(\frac{\theta_{1}+\theta_{2}}{2}\right) \leq \theta<\theta_{2} \Rightarrow \sigma_{3}(\theta)^{*}>0
\end{aligned}
$$

Let us now consider the face whose normal unit is $\vec{n}_{2}$ in which the magnetic pole surface density is defined by $J \cos \left(\frac{\theta_{1}-\theta_{2}}{2}\right)$. We call this face $f_{2}$. We deduct directly that

$$
\forall \theta \in f_{2} \Rightarrow \sigma_{2}^{*}>0
$$

By the same token, for the face whose normal unit is $\vec{n}_{4}$ in which the magnetic pole surface density is defined by $-J \cos \left(\frac{\theta_{1}-\theta_{2}}{2}\right)$ and by using the notation $f_{4}$ for this face, we deduct that:

$$
\forall \theta \in f_{4} \Rightarrow \sigma_{4}^{*}<0
$$

By using the mathematical properties determined previously, we can display in Fig. 4 the repartition of the magnetic poles.

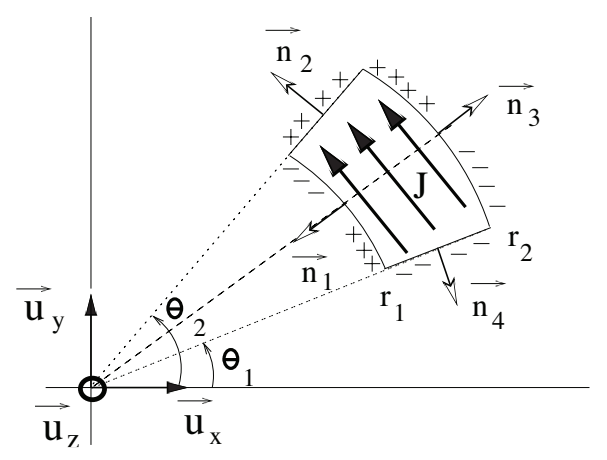

Figure 4. Representation of the fictitious magnetic poles on the faces of the tile permanent magnet whose polarization is both uniform and radial. 
Progress In Electromagnetics Research B, Vol. 13, 2009

\section{EXPRESSION OF THE THREE COMPONENTS $\boldsymbol{H}_{r}$, $H_{\theta}, H_{z}$}

These three components are functions of the parameters $r, \theta$ and $z$.

\subsection{Basic Equation}

The magnetic field created by a tile permanent magnet whose polarization is both uniform and tangential can be determined by using the Coulombian model. By definition, the magnetic field $\mathbf{H}(r, \theta, z)$ created by this magnet is expressed as follows:

$$
\begin{aligned}
\mathbf{H}(r, \theta, z) & =\iint_{S_{1}} \frac{\sigma_{1}(\tilde{\theta})^{*}}{4 \pi \mu_{0}} \frac{\overrightarrow{u_{1}}}{\left|\overrightarrow{u_{1}}\right|^{3}} d S_{1} \\
+ & \iint_{S_{2}} \frac{\sigma_{2}^{*}}{4 \pi \mu_{0}} \frac{\overrightarrow{u_{2}}}{\left|\overrightarrow{u_{2}}\right|^{3}} d S_{2} \\
+ & \iint_{S_{3}} \frac{\sigma_{3}(\tilde{\theta})^{*}}{4 \pi \mu_{0}} \frac{\overrightarrow{u_{3}}}{\left|\overrightarrow{u_{3}}\right|^{3}} d S_{3} \\
& +\iint_{S_{4}} \frac{\sigma_{4}^{*}}{4 \pi \mu_{0}} \frac{\overrightarrow{u_{4}}}{\left|\overrightarrow{u_{4}}\right|^{3}} d S_{4}
\end{aligned}
$$

where $\vec{u}_{i}$ is the vector between the observation point and a point owing to the surface $S_{i}$.

It is noted that $\vec{u}_{1}, \vec{u}_{2}, \vec{u}_{3}$ and $\vec{u}_{4}$ are expressed in a general form because we are interested in a three-dimensional solution for calculating the magnetic field for all points in space. The integration of (14) leads to the three magnetic field components along the three defined axes: $H_{r}(r, \theta, z), H_{\theta}(r, \theta, z)$ and $H_{z}(r, \theta, z)$.

\subsection{Radial Component}

The radial component of the magnetic field created by a tile permanent

magnet whose polarization is both uniform and tangential can be expressed as follows:

$$
\begin{aligned}
H_{r}(r, \theta, z)= & \sum_{i=1}^{2} \sum_{j=1}^{2}(-1)^{(i+j)} h_{r}^{(I)}\left(r_{i}, z_{j}\right) \\
& +\sum_{i=1}^{2} \sum_{j=1}^{2} \sum_{k=1}^{2}(-1)^{(i+j+k)} h_{r}^{(I I)}\left(r_{i}, z_{j}, \theta_{k}\right)
\end{aligned}
$$


where $h_{r}^{(I)}\left(r_{i}, z_{j}\right)$ represents the magnetic field created by the fictitious magnetic poles located on the arc-shaped faces of the tile permanent magnet and $h_{r}^{(I I)}\left(r_{i}, z_{j}, \theta_{k}\right)$ represents the magnetic field created by the fictitious magnetic poles located on the straight faces of the tile permanent magnet.

$$
\begin{aligned}
h_{r}^{(I)}\left(r_{i}, z_{j}\right)= & \frac{J}{4 \pi \mu_{0}} r_{i}\left(z-z_{j}\right) \tilde{\mathbf{E}^{*}}\left[\theta_{a}, r_{i}, z_{j}\right] \\
h_{r}^{(I I)}\left(r_{i}, z_{j}, \theta_{k}\right)= & -\frac{J \cos \left(\frac{\theta_{1}-\theta_{2}}{2}\right)}{8 \pi \mu_{0} X_{k}}\left(\left(1-x_{k}^{2}+x_{k} X_{k}\right) \log \left[A_{i, j, k}\right]\right) \\
& -\frac{J \cos \left(\frac{\theta_{1}-\theta_{2}}{2}\right)}{8 \pi \mu_{0} X_{k}}\left(\left(-1+x_{k}^{2}+x_{k} X_{k}\right) \log \left[B_{i, j, k}\right]\right)
\end{aligned}
$$

where $\tilde{\mathbf{E}^{*}}\left[\theta_{a}, r_{i}, z_{j}\right]$ can be seen as a non-classical elliptic integral that is defined as follows:

$$
\tilde{\mathbf{E}^{*}}\left[\theta_{a}, r_{i}, z_{j}\right]=\int_{\theta_{1}}^{\theta_{2}} \frac{\left(r-r_{i} \cos (\theta-\tilde{\theta})\right) \sin \left(\theta_{a}-\tilde{\theta}\right)}{\xi(i, j, \tilde{\theta})\left(\xi(i, j, \tilde{\theta})^{2}-\left(z-z_{j}\right)^{2}\right)} d \tilde{\theta}
$$

and

$$
\begin{aligned}
A_{i, j, k}= & \frac{2\left(r r_{i} X_{k}^{2}+r^{2} X_{k}^{2}\left(-x_{k}+X_{k}\right)\right)}{\left(-X_{k}^{2}+x_{x} X_{k}\right)\left(r_{i}+r\left(-x_{k}+X_{k}\right)\right)\left(z-z_{j}\right)} \\
& -\frac{2 X_{k}\left(z-z_{j}+\sqrt{r^{2}+r_{i}^{2}-2 r r_{i} x_{k}+\left(z-z_{j}\right)^{2}}\right)}{\left(-X_{k}^{2}+x_{x} X_{k}\right)\left(r_{i}+r\left(-x_{k}+X_{k}\right)\right)} \\
B_{i, j, k}= & -\frac{2\left(r r_{i} X_{k}^{2}-r^{2} X_{k}^{2}\left(x_{k}+X_{k}\right)\right)}{\left(X_{k}^{2}+x_{k} X_{k}\right)\left(r_{i}-r\left(x_{k}+X_{k}\right)\right)\left(z-z_{j}\right)} \\
& +\frac{2 X_{k}\left(z-z_{j}+\sqrt{r^{2}+r_{i}^{2}-2 r r_{i} x_{k}+\left(z-z_{j}\right)^{2}}\right)}{\left(X_{k}^{2}+x_{k} X_{k}\right)\left(r_{i}-r\left(x_{k}+X_{k}\right)\right)}
\end{aligned}
$$

with

$$
X_{k}=\sqrt{x_{k}^{2}-1}=\sqrt{\cos \left(\theta-\theta_{k}\right)^{2}-1}
$$

and

$$
\xi\left(i, j, \theta_{k}\right)=\sqrt{r^{2}+r_{i}^{2}+\left(z-z_{j}\right)^{2}-2 r r_{i} \cos \left(\theta-\theta_{k}\right)}
$$

It is emphasized here that $h_{r}^{(I I)}\left(r_{i}, z_{j}, \theta_{k}\right)$ is fully analytical whereas $h_{r}^{(I)}\left(r_{i}, z_{j}\right)$ is based on a non-classical elliptic integral. 
It has to be noted that all the numerical calculations to illustrate the analytical formulations will be done in this section for the following tile dimensions: $r_{1}=0.025 \mathrm{~m}, r_{2}=0.028 \mathrm{~m}, z_{2}-z_{1}=0.003 \mathrm{~m}$, $\theta_{2}-\theta_{1}=\frac{\pi}{6} \mathrm{rad}$. Moreover, the polarization is $J=1 \mathrm{~T}$ and the observation path always corresponds to: $z=0.001 \mathrm{~m}, r=0.024 \mathrm{~m}$.

Figure 5 represents the radial component versus the angle $\theta$ for the defined set of values.

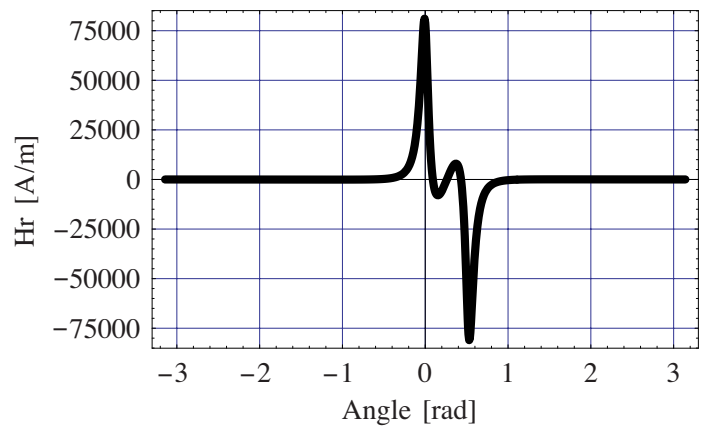

Figure 5. Representation of the radial component $H_{r}$ of the magnetic field created by a tile permanent magnet whose polarization is both uniform and tangential. We take $r_{1}=0.025 \mathrm{~m}, r_{2}=0.028 \mathrm{~m}$, its angular width is $\theta_{2}-\theta_{1}=\frac{\pi}{6} \mathrm{rad}, J=1 \mathrm{~T}, z=0.001 \mathrm{~m}, r=0.024 \mathrm{~m}$, $z_{2}-z_{1}=0.003 \mathrm{~m}$.

\subsection{Azimuthal Component}

The azimuthal component of the magnetic field created by a tile permanent magnet whose polarization is both uniform and tangential is expressed as follows:

$$
\begin{aligned}
H_{\theta}(r, \theta, z)= & \sum_{i=1}^{2} \sum_{j=1}^{2}(-1)^{(i+j)} h_{\theta}^{(I)}\left(r_{i}, z_{j}\right) \\
& +\sum_{i=1}^{2} \sum_{j=1}^{2} \sum_{k=1}^{2}(-1)^{(1+i+j+k)} h_{\theta}^{(I I)}\left(r_{i}, z_{j}, \theta_{k}\right)
\end{aligned}
$$

with

$$
h_{\theta}^{(I)}\left(r_{i}, z_{j}\right)=\frac{J}{4 \pi \mu_{0}} r_{i}^{2}\left(z_{j}-z\right) \tilde{\mathbf{L}^{*}}\left[\frac{\theta_{1}+\theta_{2}}{2}, r_{i}, z_{j}\right]
$$


where $\tilde{\mathbf{L}^{*}}\left[\theta_{a}, r_{i}, z_{j}\right]$ can be seen as a non classical definite elliptic integral.

$$
\tilde{\mathbf{L}^{*}}\left[\theta_{a}, r_{i}, z_{j}\right]=\int_{\theta_{1}}^{\theta_{2}} \frac{\sin \left(\theta_{a}-\tilde{\theta}\right) \sin (\theta-\tilde{\theta})}{\xi(i, j, \tilde{\theta})\left(\xi(i, j, \tilde{\theta})^{2}-\left(z-z_{j}\right)^{2}\right)} d \tilde{\theta}
$$

and

$$
\begin{aligned}
h_{\theta}^{(I I)}\left(r_{i}, z_{j}, \theta_{k}\right)= & \frac{J \cos \left(\frac{\theta_{1}-\theta_{2}}{2}\right)}{8 \pi \mu_{0}} \frac{2 r+y_{k}\left(-x_{k}+\tilde{X}_{k}\right)}{\tilde{X}_{k}} \log \left[C_{i, j, k}\right] \\
& +\frac{J \cos \left(\frac{\theta_{1}-\theta_{2}}{2}\right)}{8 \pi \mu_{0}} \frac{-2 r+y_{k}\left(x_{k}+\tilde{X}_{k}\right)}{\tilde{X}_{k}} \log \left[D_{i, j, k}\right] \\
C_{i, j, k}= & \frac{4 \tilde{X}_{k}\left(\left(z_{j}-z\right)-\sqrt{r^{2}+r_{i}^{2}-r_{i} \tilde{x_{k}}+\left(z-z_{j}\right)^{2}}\right)}{\left(2 r_{i}-\tilde{x_{k}}+\tilde{X}_{k}\right)\left(2 r+\left(X_{k}-\tilde{x_{k}}\right) y_{k}\right)} \\
& +\frac{\tilde{X}_{k}\left(\tilde{x_{k}}-4 r^{2}\right)+\left(2 r_{i}-\tilde{x_{k}}\right)\left(\tilde{x_{k}}-4 r^{2}\right)}{\left(2 r_{i}-\tilde{x_{k}}+\tilde{X}_{k}\right)\left(2 r+\left(X_{k}-\tilde{x_{k}}\right) y_{k}\right)\left(z-z_{j}\right)} \\
D_{i, j, k}= & \frac{4 \tilde{X}_{k}\left(\sqrt{r^{2}+r_{i}^{2}-r_{i} \tilde{x_{k}}+\left(z-z_{j}\right)^{2}}+\left(z-z_{j}\right)\right)}{\left(-2 r_{i}+\tilde{x_{k}}+\tilde{X}_{k}\right)\left(-2 r+\left(\tilde{x_{k}}+\tilde{X}_{k}\right) y_{k}\right)} \\
& +\frac{\left(4 r^{2}-\tilde{x}^{2}\right)}{\left(-2 r+\left(\tilde{x_{k}}+\tilde{X}_{k}\right) y_{k}\right)\left(z-z_{j}\right)}
\end{aligned}
$$

with

$$
\begin{aligned}
\tilde{X}_{k}=\sqrt{{\tilde{x_{k}}}^{2}-4 r^{2}} & =\sqrt{\left(2 r \cos \left(\theta-\theta_{k}\right)\right)^{2}-4 r^{2}} \\
y_{k} & =\sin \left(\theta-\theta_{k}\right)
\end{aligned}
$$

Figure 6 represents this radial component versus the angle $\theta$ with the defined values of parameters. 


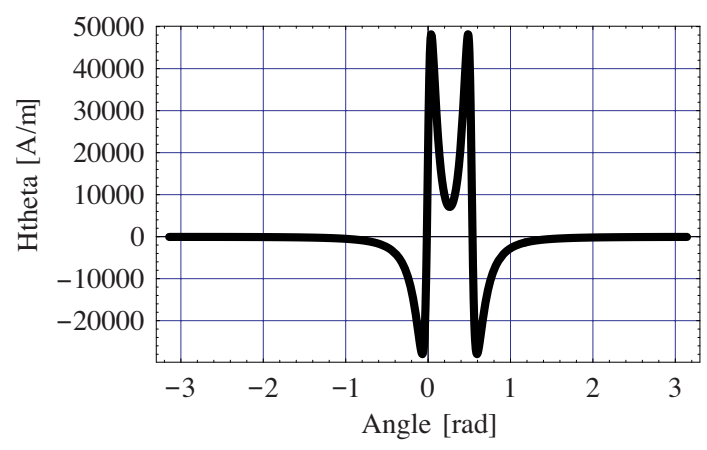

Figure 6. Representation of the azimuthal component $H_{\theta}$ of the magnetic field created by a tile permanent magnet whose polarization is both uniform and tangential. We take $r_{1}=0.025 \mathrm{~m}, r_{2}=0.028 \mathrm{~m}$, its angular width is $\theta_{2}-\theta_{1}=\frac{\pi}{6} \mathrm{rad}, J=1 \mathrm{~T}, z=0.001 \mathrm{~m}, r=0.029 \mathrm{~m}$, $z_{2}-z_{1}=0.003 \mathrm{~m}$.

\subsection{Axial Component}

The axial component $H_{z}(r, \theta, z)$ can be expressed as follows:

$$
\begin{aligned}
H_{z}(r, \theta, z)= & \sum_{i=1}^{2} \sum_{j=1}^{2}(-1)^{(i+j)} h_{z}^{(I)}\left(r_{i}, z_{j}\right) \\
& +\sum_{i=1}^{2} \sum_{j=1}^{2} \sum_{k=1}^{2}(-1)^{(i+j+k)} h_{z}^{(I I)}\left(r_{i}, z_{j}, \theta_{k}\right)
\end{aligned}
$$

with

$$
h_{z}^{(I)}\left(r_{i}, z_{j}\right)=\frac{J}{4 \pi \mu_{0}}\left(-r_{i}\right) \tilde{\mathbf{K}}^{*}\left[\frac{\theta_{1}+\theta_{2}}{2}, r_{i}, z_{j}\right]
$$

where $\tilde{\mathbf{K}}^{*}\left[\theta_{a}, r_{i}, z_{j}\right]$ represents a non-classical definite elliptic integral that is defined as follows:

$$
\tilde{\mathbf{K}}^{*}\left[\theta_{a}, r_{i}, z_{j}\right]=\int_{\theta_{1}}^{\theta_{2}} \frac{\sin \left(\theta_{a}-\tilde{\theta}\right)}{\xi(i, j, \tilde{\theta})} d \tilde{\theta}
$$

and

$$
h_{z}^{(I I)}\left(r_{i}, z_{j}, \theta_{k}\right)=\frac{J}{4 \pi \mu_{0}} \cos \left(\frac{\theta_{1}-\theta_{2}}{2}\right) \log \left[r_{i}-r \cos \left(\theta-\theta_{k}\right)+\xi\left(i, j, \theta_{k}\right)\right]
$$




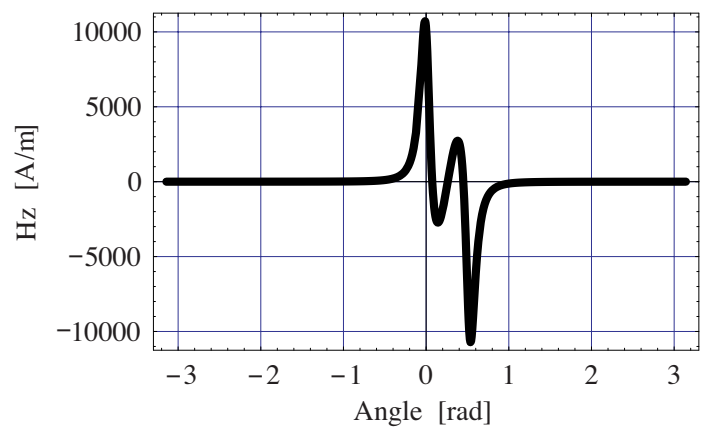

Figure 7. Representation of the axial component $H_{z}$ of the magnetic field created by a tile permanent magnet whose polarization is both uniform and tangential. We take $r_{1}=0.025 \mathrm{~m}, r_{2}=0.028 \mathrm{~m}$, $\theta_{2}-\theta_{1}=\frac{\pi}{6} \mathrm{rad}, J=1 \mathrm{~T}, z=0.001 \mathrm{~m}, r=0.024 \mathrm{~m}, z_{2}-z_{1}=0.003 \mathrm{~m}$.

Figure 7 represents this radial component versus the angle $\theta$ with the defined values of parameters.

\section{INFLUENCE OF THE TILE ANGULAR WIDTH ON THE THREE MAGNETIC FIELD COMPONENTS}

We have seen in the previous section that the three components of the magnetic field created by a tile permanent magnet whose polarization is both uniform and tangential can be determined by using a threedimensional approach. However, the contributions of all the surfaces densities have not the same weight but depend greatly on the tile permanent magnet dimensions. Indeed, the scalar products between the polarization vector $\vec{J}$ and the normal units $\vec{n}_{1}$ and $\vec{n}_{3}$ tend to zero when the curvature of the inner and outer faces of the tile permanent magnet becomes negligible or when the angular width tends to zero. We illustrate this phenomenon in this section by considering an alternate magnet structure in which we use 6,8 or 12 tile permanent magnets.

\subsection{Radial Component}

First, we study the radial field produced by an alternate magnet structure using respectively 6,8 and 12 tile permanent magnets whose polarizations are both uniform and tangential. We represent in Figs. 8, 9 and 10 the radial field versus the angle $\theta$ in these three configurations. The numerical set of parameters is the same, except for the angular 


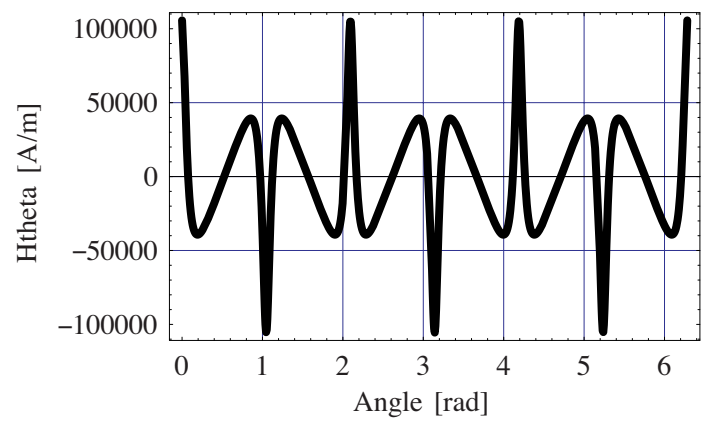

Figure 8. Representation of the radial component $H_{r}$ of the magnetic field created by an alternate magnet structure owing 6 tile permanent magnets whose polarization is both uniform and tangential. We take $r_{1}=0.025 \mathrm{~m}, r_{2}=0.028 \mathrm{~m}, \theta_{2}-\theta_{1}=\frac{\pi}{3} \mathrm{rad}, J=1 \mathrm{~T}, z=0.001 \mathrm{~m}$, $r=0.024 \mathrm{~m}, z_{2}-z_{1}=0.003 \mathrm{~m}$.

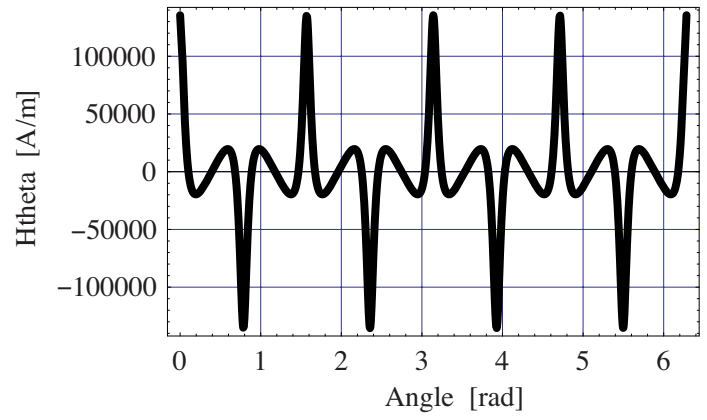

Figure 9. Representation of the radial component $H_{r}$ of the magnetic field created by an alternate magnet structure owing 8 tile permanent magnets whose polarization is both uniform and tangential. We take $r_{1}=0.025 \mathrm{~m}, r_{2}=0.028 \mathrm{~m}, \theta_{2}-\theta_{1}=\frac{\pi}{4} \mathrm{rad}, J=1 \mathrm{~T}, z=0.001 \mathrm{~m}$, $r=0.024 \mathrm{~m}, z_{2}-z_{1}=0.003 \mathrm{~m}$.

width which depends on the tile number and will be specified in each case. Figs. 8, 9 and 10 clearly show that the effects of the magnetic pole surface densities located on the inner and outer faces of the tile permanent magnet become negligible when the angular width of the tile permanent magnet decreases. This is in fact an interesting point because it gives indications about the number of tile permanent magnets that should be used in ironless structures. Indeed, if a great radial field is required, it is more interesting to use 12 tiles rather than 6 


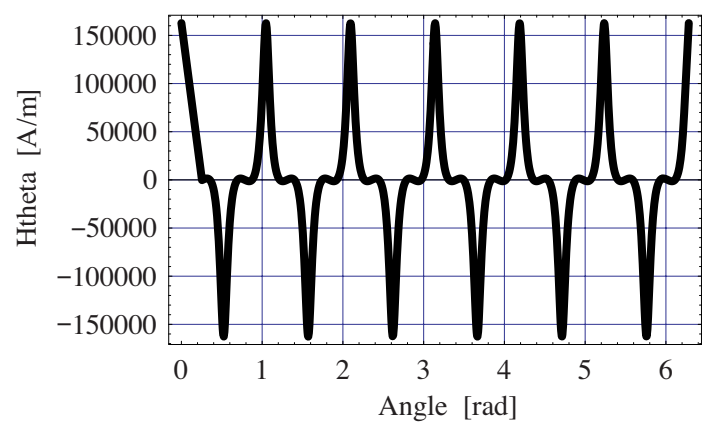

Figure 10. Representation of the radial component $H_{r}$ of the magnetic field created by an alternate magnet structure owing 12 tile permanent magnets whose polarization is both uniform and tangential. We take $r_{1}=0.025 \mathrm{~m}, r_{2}=0.028 \mathrm{~m}, \theta_{2}-\theta_{1}=\frac{\pi}{6} \mathrm{rad}, J=1 \mathrm{~T}$, $z=0.001 \mathrm{~m}, r=0.024 \mathrm{~m}, z_{2}-z_{1}=0.003 \mathrm{~m}$.

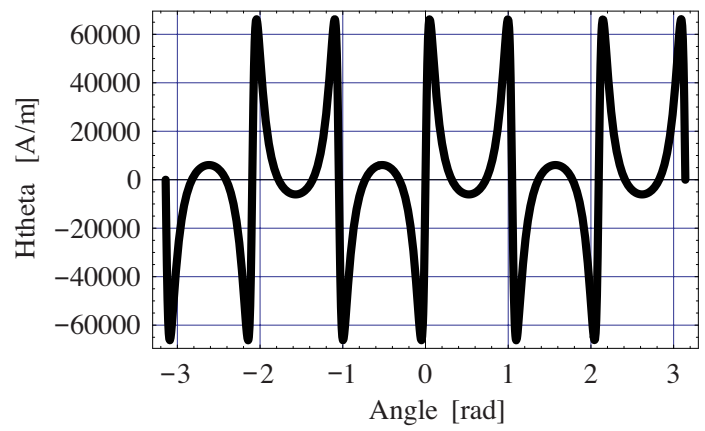

Figure 11. Representation of the azimuthal component $H_{\theta}$ of the magnetic field created by an alternate magnet structure owing 6 tile permanent magnets whose polarization is both uniform and tangential. We take $r_{1}=0.025 \mathrm{~m}, r_{2}=0.028 \mathrm{~m}, \theta_{2}-\theta_{1}=\frac{\pi}{3} \mathrm{rad}, J=1 \mathrm{~T}$, $z=0.001 \mathrm{~m}, r=0.024 \mathrm{~m}, z_{2}-z_{1}=0.003 \mathrm{~m}$.

tiles. However, it must be emphasized here that a small tile permanent magnet is generally more difficult to manufacture than a greater one.

\subsection{Azimuthal Component}

Second, we study now the azimuthal field produced by an alternate magnet structure using respectively 6,8 and 12 tile permanent magnets whose polarizations are both uniform and tangential. We represent in Figs. 11, 12 and 13 the azimuthal field versus the angle $\theta$ in these three configurations. 


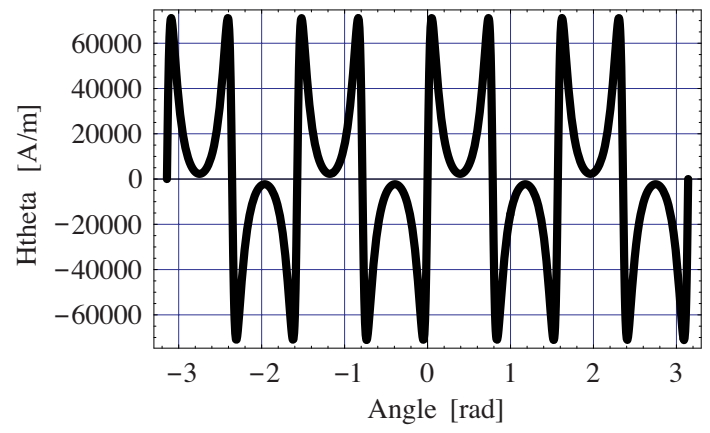

Figure 12. Representation of the azimuthal component $H_{\theta}$ of the magnetic field created by an alternate magnet structure owing 8 tile permanent magnets whose polarization is both uniform and tangential. We take $r_{1}=0.025 \mathrm{~m}, r_{2}=0.028 \mathrm{~m}, \theta_{2}-\theta_{1}=\frac{\pi}{4} \mathrm{rad}, J=1 \mathrm{~T}$, $z=0.001 \mathrm{~m}, r=0.024 \mathrm{~m}, z_{2}-z_{1}=0.003 \mathrm{~m}$.

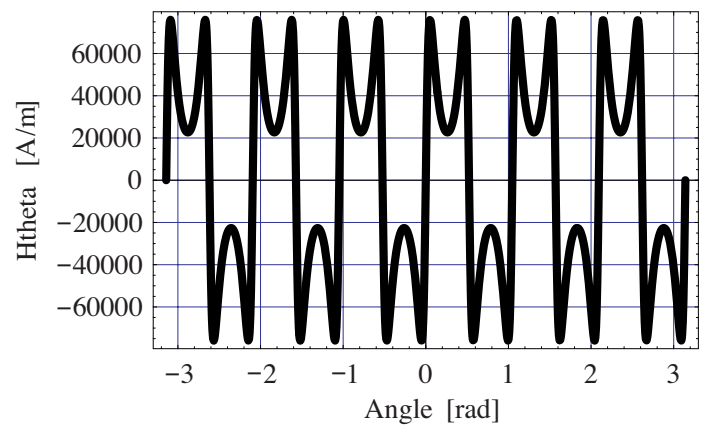

Figure 13. Representation of the azimuthal component $H_{z}$ of the magnetic field created by an alternate magnet structure owing 6 tile permanent magnets whose polarization is both uniform and tangential. We take $r_{1}=0.025 \mathrm{~m}, r_{2}=0.028 \mathrm{~m}, \theta_{2}-\theta_{1}=\frac{\pi}{3} \mathrm{rad}, J=1 \mathrm{~T}$, $z=0.001 \mathrm{~m}, r=0.024 \mathrm{~m}, z_{2}-z_{1}=0.003 \mathrm{~m}$.

Here again, Figs. 11, 12 and 13 clearly show that the effects of the magnetic pole surface densities located on the inner and outer faces of the tile permanent magnet become negligible when the angular width of the tile permanent magnet decreases. In addition, the number of tile permanent magnets has also an influence on the shape of the azimuthal field. Consequently, the tile permanent magnet dimension should also be optimized according to the intended application (in which a given azimuthal field shape is required). 


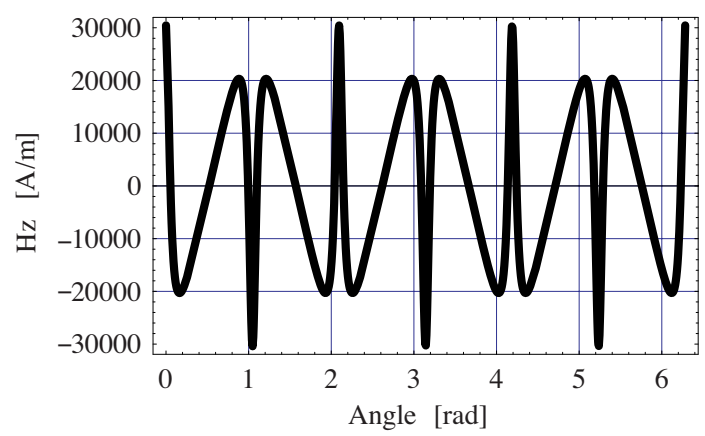

Figure 14. Axial component $H_{z}$. Alternate structure with 6 tiles. Tile: $r_{1}=0.025 \mathrm{~m}, r_{2}=0.028 \mathrm{~m}, z_{2}-z_{1}=0.003 \mathrm{~m}, \theta_{2}-\theta_{1}=\frac{\pi}{3} \mathrm{rad}$, $J=1 \mathrm{~T}$. Observation path: $z=0.001 \mathrm{~m}, r=0.024 \mathrm{~m}$.

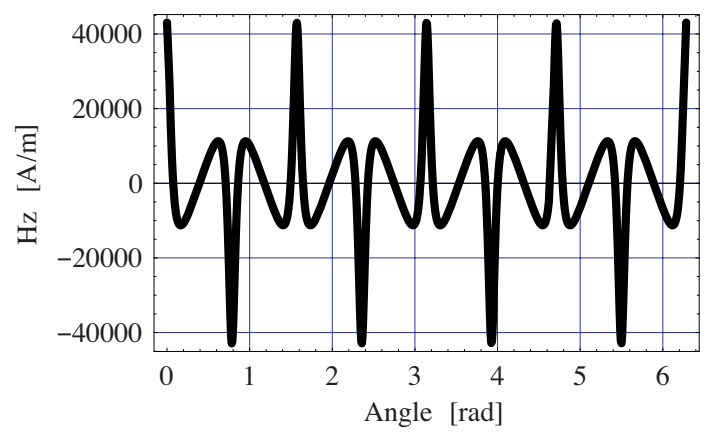

Figure 15. Representation of the axial component $H_{z}$ of the magnetic field created by an alternate magnet structure owing 8 tile permanent magnets whose polarization is both uniform and tangential. We take $r_{1}=0.025 \mathrm{~m}, r_{2}=0.028 \mathrm{~m}, \theta_{2}-\theta_{1}=\frac{\pi}{4} \mathrm{rad}, J=1 \mathrm{~T}, z=0.001 \mathrm{~m}$, $r=0.024 \mathrm{~m}, z_{2}-z_{1}=0.003 \mathrm{~m}$.

\subsection{Axial Component}

Eventually, we study the axial field produced by an alternate magnet structure using still respectively 6,8 and 12 tile permanent magnets whose polarizations are both uniform and tangential. We represent in Figs. 14, 15 and 16 the axial field versus the angle $\theta$ in these three configurations. Here again, Figs. 14, 15 and 16 clearly show that the effects of the magnetic pole surface densities located on the inner and outer faces of the tile permanent magnet become negligible when the angular width of the tile permanent magnet decreases. In addition, the number of tile permanent magnets has also an influence on the 


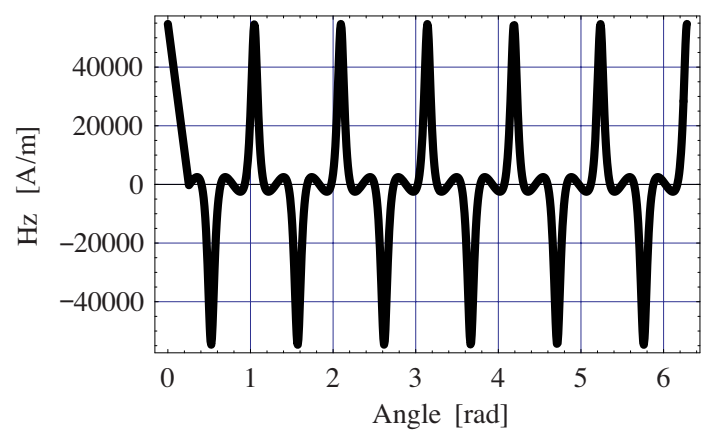

Figure 16. Representation of the axial component $H_{z}$ of the magnetic field created by an alternate magnet structure owing 12 tile permanent magnets whose polarization is both uniform and tangential. We take $r_{1}=0.025 \mathrm{~m}, r_{2}=0.028 \mathrm{~m}, \theta_{2}-\theta_{1}=\frac{\pi}{6} \mathrm{rad}, J=1 \mathrm{~T}, z=0.001 \mathrm{~m}$, $r=0.024 \mathrm{~m}, z_{2}-z_{1}=0.003 \mathrm{~m}$.

shape of the axial field. For example, Fig. 14 shows that the use of only 6 permanent magnets in an ironless structure whose diameter is small (here $0.05 \mathrm{~m}$ ) is not sufficient. Thus, our three-dimensional approach allows us to optimize easily magnet dimensions for studying the magnetic field produced by such structures.

\section{CONCLUSION}

This paper has presented 3D analytical expressions for studying the magnetic field created by a tile permanent magnet whose polarization is both uniform and tangential. The three magnetic components of this magnetic field are determined by using the Colombian model. As no simplifying assumptions are used for calculating the radial, axial and azimuthal fields created by a tile permanent magnet, our expressions are valid for all points in space, whatever the magnet dimensions. We also discuss the influence of the angular width of a tile permanent magnet on the field produced. The results obtained confirm that the scalar product $\vec{J} \cdot \overrightarrow{n_{i}}$ where $\vec{n}_{i}$ is the normal unit of the face $i$ can be a good indicator for studying the effects of all the magnetic pole charge contributions on the magnetic field created. The expressions given in this paper are available online [35] and for each expression, a numerical calculation has been carried out for confirming our three-dimensional approach. 


\section{REFERENCES}

1. Ravaud, R., G. Lemarquand, V. Lemarquand, and C. Depollier, "Improvement of the analytical calculation of the magnetic field produced by permanent magnet rings," Progress In Electromagnetics Research, PIER 88, 307-319, 2008.

2. Babic, S. and C. Akyel, "Improvement of the analytical calculation of the magnetic field produced by permanent magnet rings," Progress In Electromagnetics Research C, Vol. 5, 71-82, 2008.

3. Furlani, E. P., Permanent Magnet and Electromechanical Devices: Materials, Analysis and Applications, Academic Press, 2001.

4. Ravaud, R., G. Lemarquand, V. Lemarquand, and C. Depollier, "Analytical calculation of the magnetic field created by permanent-magnet rings," IEEE Trans. Magn., Vol. 44, No. 8, 1982-1989, 2008.

5. Ravaud, R., G. Lemarquand, V. Lemarquand, and C. Depollier, "Discussion about the analytical calculation of the magnetic field created by permanent magnets," Progress In Electromagnetics Research B, Vol. 11, 281-297, 2009.

6. Selvaggi, J. P., S. Salon, O. M. Kwon, and M. Chari, "Computation of the three-dimensional magnetic field from solid permanent-magnet bipolar cylinders by employing toroidal harmonics," IEEE Trans. Magn., Vol. 43, No. 10, 3833-3839, 2007.

7. Azzerboni, B. and G. Saraceno, "Three-dimensional calculation of the magnetic field created by current-carrying massive disks," IEEE Trans. Magn., Vol. 34, No. 5, 2601-2604, 1998.

8. Azzerboni, B. and E. Cardelli, "Magnetic field evaluation for disk conductors," IEEE Trans. Magn., Vol. 29, No. 6, 2419-2421, 1993.

9. Azzerboni, B., E. Cardelli, M. Raugi, A. Tellini, and G. Tina, "Magnetic field evaluation for thick annular conductors," IEEE Trans. Magn., Vol. 29, No. 3, 2090-2094, 1993.

10. Rakotoarison, H. L., J. P. Yonnet, and B. Delinchant, "Using colombian approach for modeling scalar potential and magnetic field of a permanent magnet with radial polarization," IEEE Trans. Magn., Vol. 43, No. 4, 1261-1264, 2007.

11. Durand, E., Electrostatique, Vol. 1, 248-251, Masson Editeur, Paris, France, 1964.

12. Babic, S. and C. Akyel, "Magnetic force calculation between thin coaxial circular coils in air," IEEE Trans. Magn., Vol. 44, No. 4, 445-452, 2008.

13. Babic, S., C. Akyel, and S. Salon, "New procedures for calculating 
the mutual inductance of the system: Filamentary circular coilmassive circular solenoid," IEEE Trans. Magn., Vol. 39, No. 3, 1131-1134, 2003.

14. Babic, S., C. Akyel, S. Salon, and S. Kincic, "New expressions for calculating the magnetic field created by radial current in massive disks," IEEE Trans. Magn., Vol. 38, No. 2, 497-500, 2002.

15. Babic, S., S. Salon, and C. Akyel, "The mutual inductance of two thin coaxial disk coils in air," IEEE Trans. Magn., Vol. 40, No. 2, 822-825, 2004.

16. Conway, J., "Noncoaxial inductance calculations without the vector potential for axisymmetric coils and planar coils," IEEE Trans. Magn., Vol. 44, No. 10, 453-462, 2008.

17. Furlani, E. P., S. Reznik, and A. Kroll, "A three-dimensional field solution for radially polarized cylinders," IEEE Trans. Magn., Vol. 31, No. 1, 844-851, 1995.

18. Furlani, E. P., "Field analysis and optimization of NDFEB axial field permanent magnet motors," IEEE Trans. Magn., Vol. 33, No. 5, 3883-3885, 1997.

19. Furlani, E. P. and M. Knewston, "A three-dimensional field solution for permanent-magnet axial-field motors," IEEE Trans. Magn., Vol. 33, No. 1, 2322-2325, 1997.

20. Furlani, E. P., "A two-dimensional analysis for the coupling of magnetic gears," IEEE Trans. Magn., Vol. 33, No. 3, 2317-2321, 1997.

21. Mayergoyz, D. and E. P. Furlani, "The Computation of magnetic fields of permanent magnet cylinders used in the electrophotographic process," J. Appl. Phys., Vol. 73, No. 10, 5440-5442, 1993.

22. Yonnet, J. P., "Passive magnetic bearings with permanent magnets," IEEE Trans. Magn., Vol. 14, No. 5, 803-805, 1978.

23. Abele, M., J. Jensen, and H. Rusinek, "Generation of uniform high fields with magnetized wedges," IEEE Trans. Magn., Vol. 33, No. 5, 3874-3876, 1997.

24. Aydin, M., Z. Zhu, T. Lipo, and D. Howe, "Minimization of cogging torque in axial-flux permanent-magnet machines: Design concepts," IEEE Trans. Magn., Vol. 43, No. 9, 3614-3622, 2007.

25. Marinescu, M. and N. Marinescu, "Compensation of anisotropy effects in flux-confining permanent-magnet structures," IEEE Trans. Magn., Vol. 25, No. 5, 3899-3901, 1989.

26. Akoun, G. and J. P. Yonnet, "3d analytical calculation of the forces exerted between two cuboidal magnets," IEEE Trans. 
Magn., Vol. 20, No. 5, 1962-1964, 1984.

27. Yong, L., Z. Jibin, and L. Yongping, "Optimum design of magnet shape in permanent-magnet synchronous motors," IEEE Trans. Magn., Vol. 39, No. 11, 3523-4205, 2003.

28. Lemarquand, G. and V. Lemarquand, "Annular magnet position sensor," IEEE. Trans. Magn., Vol. 26, No. 5, 2041-2043, 1990.

29. Yonnet, J. P., "Permanent magnet bearings and couplings," IEEE Trans. Magn., Vol. 17, No. 1, 1169-1173, 1981.

30. Zhu, Z. and D. Howe, "Analytical prediction of the cogging torque in radial-field permanent magnet brushless motors," IEEE Trans. Magn., Vol. 28, No. 2, 1371-1374, 1992.

31. Wang, J., G. W. Jewell, and D. Howe, "Design optimisation and comparison of permanent magnet machines topologies," IEE Proc. Elect. Power Appl., Vol. 148, 456-464, 2001.

32. Yonnet, J. P., Rare-earth Iron Permanent Magnets, Ch. Magnetomechanical devices, Oxford Science Publications, 1996.

33. Blache, C. and G. Lemarquand, "New structures for linear displacement sensor with hight magnetic field gradient," IEEE Trans. Magn., Vol. 28, No. 5, 2196-2198, 1992.

34. Halbach, K., "Design of permanent multiple magnets with oriented rec material," Nucl. Inst. Meth., Vol. 169, 1-10, 1980.

35. http://www.univ-lemans.fr/ glemar 